\title{
Prediction of flow noise around a cylinder based on Large- Eddy Simulation and acoustic analogy method
}

\author{
Qikai Qin ${ }^{1,2,3}$, Dejiang Shang ${ }^{1,2,3}$, Yongwei Liu $^{1,2,3, *}$, and Tianyu Wang ${ }^{1,2,3}$ \\ ${ }^{1}$ Acoustic Science and Technology Laboratory, Harbin Engineering University, Harbin 150001, China \\ ${ }^{2}$ Key Laboratory of Marine Information Acquisition and Security(Harbin Engineering University), Ministry of Industry and \\ Information Technology; Harbin 150001, China \\ ${ }^{3}$ College of Underwater Acoustic Engineering, Harbin Engineering University, Harbin 150001, China
}

\begin{abstract}
In this article, a hybrid method combining large eddy simulation with acoustic analogy is presented to predict three-dimensional far field noise induced by flow around a cylinder. Firstly, the governing equation including RANS equations with shear-stress transport (SST) k- $\omega$ turbulent model is numerically solved for steady flow field by using Ansys Fluent. Transient flow field is numerically solved by LES. Then, the flow field simulation results are used to compute the flowinduced noise with the FW-H integral equation method and BEM method based on Lighthill acoustic analogy equation in Actran. Before using for flow around a cylinder, accuracy of flow turbulent model in predicting turbulent flow around a cylinder is tested by comparing with available experimental data. According on the simulation result, the characteristic of the acoustic field, noise at some special points in frequency domain, the noise radiation directivity are studied. Analysis of noise distribution and frequency spectrum curves shows that dipole source takes the dominant place in the noise around a cylinder under the conditions of this article. The flow noise around a cylinder is mainly concentrated in the low frequency range.
\end{abstract}

\section{Introduction}

As a basic structural form, cylindrical structure is very common in the fields of traffic transportation engineering, ocean engineering and fluid machinery. After flowing through the cylindrical structure, the fluid wakes alternately appear with the vortex at a certain Reynolds number. These vortex streets cause pulsating pressure on the cylindrical surface, thereby generating noise. The noise characteristics of the flow around the cylinder are one of the factors that must be considered in engineering design and the basis for noise reduction. The problem of sounding around a cylinder has been studied by scholars very early. In 1977, Revell used a wind tunnel test to study cylinders with diameters of $19 \mathrm{~mm}$ and $38 \mathrm{~mm}$, testing Mach numbers from 0.1 to 0.5 , and Reynolds numbers from 45000 to 450000 . Revell measured the Reynolds number range. The relationship between the resistance of the flow around the cylinder and the flow noise is studied by the resistance coefficient of the flow around the cylinder and the total sound pressure level[1]. This experiment has been used as a benchmark for many researchers as a calculation reference. In terms of numerical simulation, Kenneth S. Brentner and Cox have simulated the two-dimensional flow around the cylinder, and most of them use the Reynolds average method[2-3]. Osamu Inoue used a

\footnotetext{
* Corresponding author: liuyongwei3000@hrbeu.edu.cn
}

direct numerical simulation method to study the generation and propagation mechanism of twodimensional cylindrical flow noise in a uniform flow[4]. Tang used the large eddy simulation method to obtain the hydrodynamic data of the circular flow around the cylinder, and then used the solution FW-H. Equation method for predicting far-field noise [5].

In this paper, the numerical simulation of the flow noise of the cylinder is carried out. The incompressible flow is used to compare different turbulence simulation methods. The transient flow field was simulated by large eddy simulation, SST model and k-Omega model respectively. The differences of each model were compared, and the flow field simulation method suitable for flow noise calculation was selected. The sound field is predicted after the flow field is calculated, and the FW-H integral method and the boundary element method are used. Through these research works, the proposed calculation method can provide support for large-scale ship flow noise prediction.

\section{Equations and mathematics}

In this study, it is assumed that fluid is incompressible. The governing equations are the mass and momentum conservations. Using the Reynolds averaging approach, the Navier - Stokes equation can be expressed as 


$$
\begin{gathered}
\frac{\partial \mu_{i}}{\partial t}+\frac{\partial\left(\mu_{i} \mu_{j}\right)}{\partial x_{j}}=f_{i}-\frac{1}{\rho} \frac{\partial p}{\partial x_{i}}+v \frac{\partial^{2} \mu_{i}}{\partial x_{i} \partial x_{j}} \\
\frac{\partial \mu_{i}}{\partial x_{i}}=0
\end{gathered}
$$

The Lighthill acoustic analogy theory combines the flow field information with the sound field information by transforming the N-S equation to solve the problem of how to establish the sound source in the flow noise. Directly transform the N-S equation and the continuity equation to obtain the non-homogeneous wave equation

$$
\begin{gathered}
\frac{1}{c_{0}^{2}} \frac{\partial^{2} P_{a}}{\partial t^{2}}-\frac{\partial^{2} P_{a}}{\partial x_{i} \partial x_{j}}=\frac{\partial^{2} T_{i j}}{\partial x_{i} \partial x_{j}} \\
T_{i j}=\rho_{0} v_{i} v_{j}
\end{gathered}
$$

\section{Numerical simulation of flow field}

Before the flow noise is predicted, it is first necessary to accurately calculate the flow field. The steady flow field is calculated using the Realizable model in the Reynolds averaging method. For the calculation of the transient flow field, this paper compares the LES, SST model, and $\mathrm{k}$-Omega model to determine which method is more suitable for the calculation.

\subsection{Model building and computational grid}

The calculation model is a cylinder with a diameter $\mathrm{D}=$ $10 \mathrm{~mm}$. The origin of the coordinate is at the center of the bottom of the cylinder. The $\mathrm{x}$ direction represents the forward flow direction, the $y$ direction represents the lateral flow direction, and the $\mathrm{z}$ direction represents the cylindrical direction. Taking the diameter $\mathrm{D}$ of the cylinder as the characteristic scale, the scale of the verification model is $20 \mathrm{D} \times 10 \mathrm{D} \times 3.14 \mathrm{D}$, the inlet of the fluid domain is $5 \mathrm{D}$ from the center of the cylinder, the outlet is $15 \mathrm{D}$ from the cylinder, and the center of both sides is $5 \mathrm{D}$ from the center of the cylinder. The calculation domain is shown in Fig. 1.

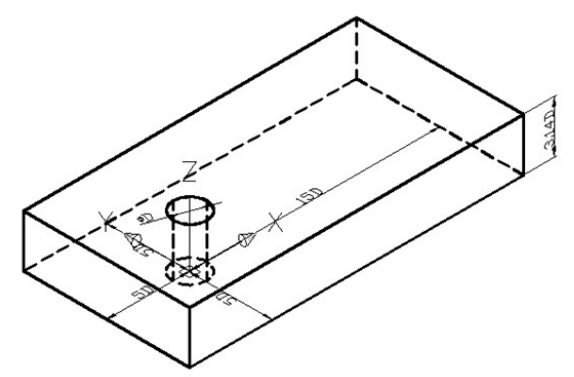

Fig. 1. Calculation domain of the flow field.

Computational grid of the fluid field is shown in Fig. 2. The flow field area is selected as a structured mesh, and the O-shaped section is divided around the cylinder. At the same time, the mesh density is gradually decreased from the cylindrical wall to the far side, thereby reducing the total mesh, to improve computing efficiency.

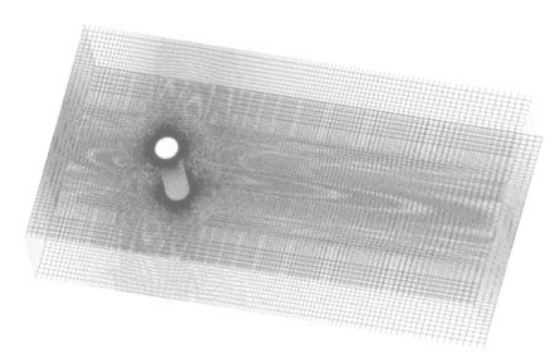

Fig. 2. Calculation grid of the flow field.

\subsection{Calculation setup}

Conditions applied to boundaries of computational domain for turbulent flow calculations, as shown in Fig. 3 , are

(1) "Velocity-inlet" condition on inlet surface in up stream flow

(2) "Pressure-outlet" condition on outlet surface in downstream flow

(3) "Free-slip-wall" condition, equivalent to "symmetry" condition in Ansys Fluent software, on side surfaces in side flow.

(4) "No slip wall" condition on cylinder surface.

The initial conditions of the flow field: the uniform flow velocity at the infinity of the flow field is $U_{0}=1 \mathrm{~m} / \mathrm{s}$, the corresponding Reynolds number is $R e=3900$, the fluid is water, the density $\rho=1000 \mathrm{~kg} / \mathrm{m}^{3}$, the dynamic viscosity coefficient $\mu=0.025641 \mathrm{~Pa} \cdot \mathrm{s}$, the reference pressure is $1 \mathrm{~atm}$, and the water temperature is $25^{\circ} \mathrm{C}$.

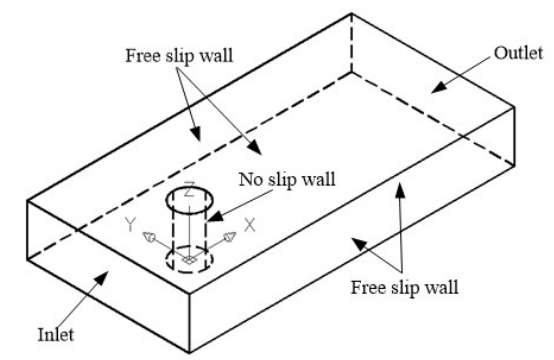

Fig. 3. Boundary conditions of the flow field.

Solver and discretization setup for governing equations are presented in Table 1.

Table 1. Solver and discretization setup.

\begin{tabular}{|c|c|}
\hline Solver type & Pressure based \\
\hline Pressure coupling & Coupled \\
\hline Gradient & Green-Gauss node based \\
\hline Momentum & Second order upwind \\
\hline Turbulent kinematic energy & Second order upwind \\
\hline
\end{tabular}

The time step determines the sampling frequency of the sound field calculation. According to Nyquist's law of sampling, the highest frequency of the sound field that can be restored is half of the sampling frequency. Assuming a maximum frequency of $8 \mathrm{kHz}$, the sampling frequency is $16 \mathrm{kHz}$, so $1 / 16000=6.25 \mathrm{e}-5 \mathrm{~s}$ is chosen as the time step for the transient calculation. 


\subsection{Simulation results}

Time history curve of the lift coefficient is shown in Fig. 4. Time history curve of the lift coefficient is shown in Fig.5. The corresponding lift coefficient curves are subjected to fast Fourier transform to obtain the vortex shedding frequency, and the corresponding Strouhal number is obtained according to the formula $S t=f D / U_{0}$. The comparison with the literature $[6,7]$ is shown in Table 2.

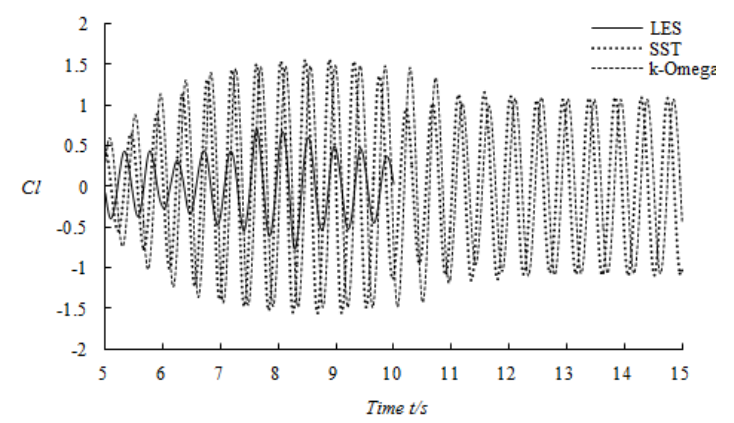

Fig. 4. Time-history diagram of the lift coefficient of a cylindrical surface.

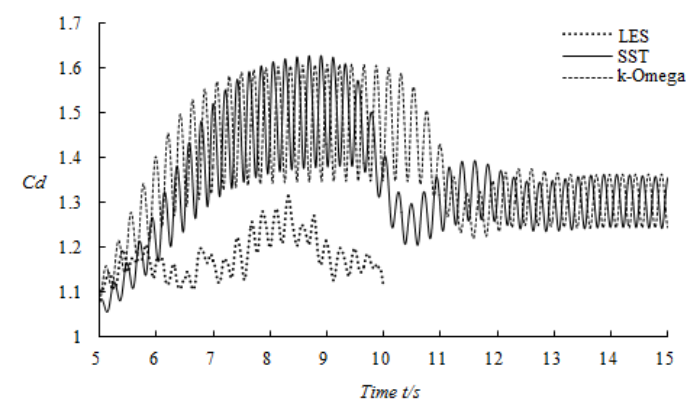

Fig. 5. Time-history diagram of the resistance coefficient of the cylindrical surface.

Table 2. Comparison of Strouhal number.

\begin{tabular}{|c|c|c|c|c|c|}
\hline & $\begin{array}{c}\text { Experimental } \\
\text { results[6] }\end{array}$ & $\begin{array}{c}\text { He’ s } \\
\text { case[7] }\end{array}$ & LES & SST & k- $\omega$ \\
\hline St & $0.215 \pm 0.005$ & 0.216 & 0.217 & 0.235 & 0.234 \\
\hline $\begin{array}{c}\text { Deviation } \\
(\%)\end{array}$ & 0 & 0.47 & 0.93 & 9.31 & 9.29 \\
\hline
\end{tabular}

Table 3. Comparison of the mean drag coefficient.

\begin{tabular}{|c|c|c|c|c|c|}
\hline & $\begin{array}{c}\text { Experimental } \\
\text { results[6] }\end{array}$ & $\begin{array}{c}\text { He' s } \\
\text { case[7] }\end{array}$ & LES & SST & k- $\omega$ \\
\hline$\overline{C_{d}}$ & $0.99 \pm 0.05$ & 1.15 & 1.17 & 1.301 & 1.333 \\
\hline $\begin{array}{c}\text { Deviation } \\
(\%)\end{array}$ & 0 & 13.9 & 12.8 & 31.41 & 34.65 \\
\hline
\end{tabular}

By comparison, it is found that for the mean drag coefficient and the Strouhal number St, the results obtained by the working condition LES are more accurate than the working conditions SST and k- $\omega$. The three turbulence models can describe the main features of vortex shedding, but the large eddy simulation (LES) is better than the k-Omega model and SST model. It indicates that the large eddy simulation method has certain advantages in the simulation of turbulence.
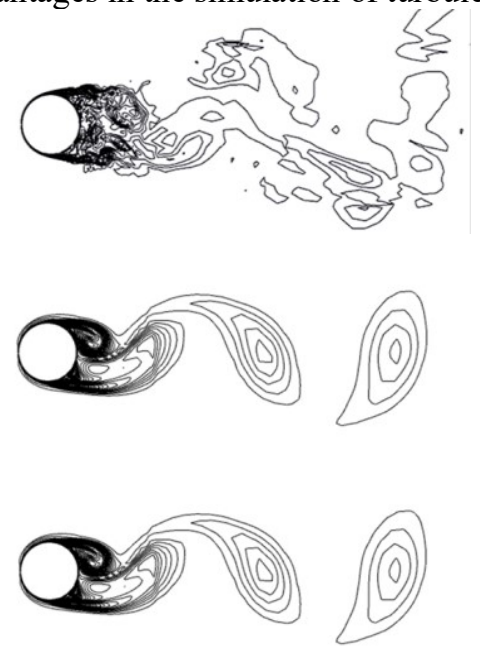

Fig. 6. Vortex contour of different turbulence model.

\section{Numerical simulation of sound field}

When the fluid flows through the cylinder, it will alternately produce the detached vortex, and at the same time form a pulsating lift on the cylindrical surface. This pulsating lift is the main cause of noise. In addition, the vortex that falls off gradually forms turbulence, which also produces quadrupole source. In this paper, a method which combines CFD and BEM is used to calculate the acoustic field.

\subsection{Calculation method and field setting}

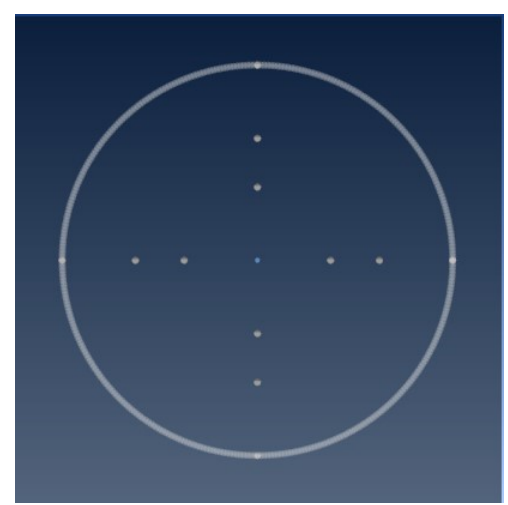

Fig. 7. Position of acoustic measurement points.

The information of the flow field pressure pulsation calculated by CFD is input into the acoustic software, and the sound field distribution in the sound source area and the sound propagation area is calculated by the BEM method. The sound pressure level and the spectrum curve of the far field are calculated by the infinite element method. The calculated mesh satisfies that the maximum mesh size greater than $1 / 6$ of the minimum acoustic wavelength. Set 12 acoustic measuring points, which are located on the horizontal and vertical lines at the center of the cylinder, and the distance from the center of the cylinder is $3 \mathrm{D}, 5 \mathrm{D}, 8 \mathrm{D}$.

\subsection{Simulation results}




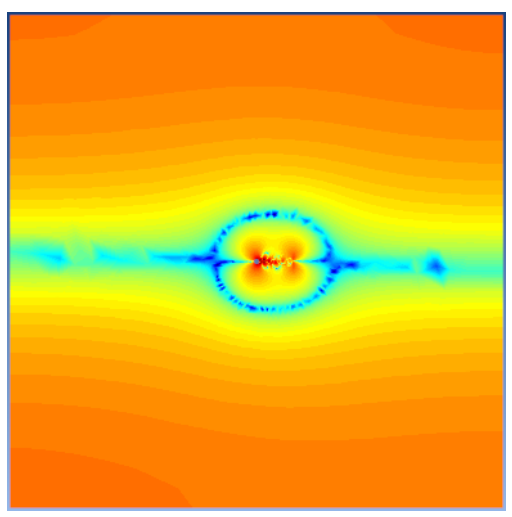

Fig. 8. Distribution contour of sound pressure at $40 \mathrm{~Hz}$.

By performing a fast Fourier transform on the sound pressure of each measurement point, the sound pressure value in the frequency domain can be obtained, and the spectral characteristic curve of the field point is as shown in Fig. 9.
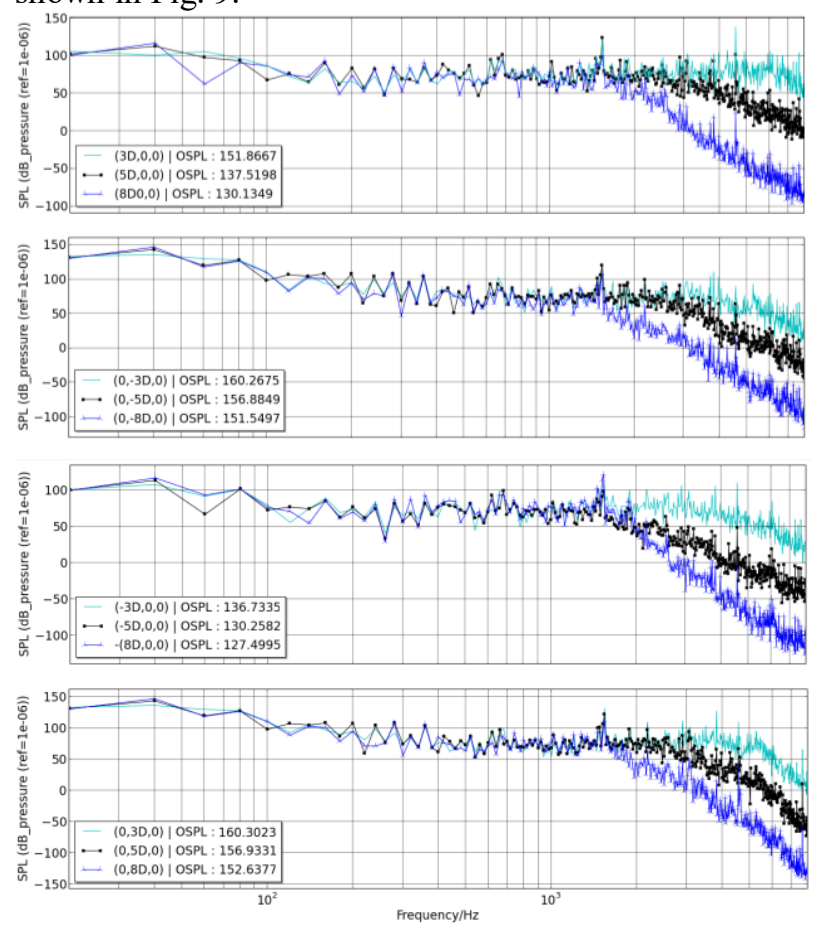

Fig. 9. Spectral characteristic curve of the field points.

By comparing the total sound pressure level curve of the measuring point, the sound pressure level of the measuring point symmetrically distributed on the upper and lower sides of the cylinder is the largest, and the sound pressure level of the measuring point decreases as the distance from the cylinder increases. The sound pressure level of the field point downstream of the cylinder is greater than the upstream. The closer to the cylinder, the greater the total sound pressure level at the measuring point. In the case where the distance from the cylinder is equal, the total sound pressure level of the measurement point in the vertical direction is larger than the horizontal direction. The total sound pressure levels of the symmetrical measurement points on both sides of the cylinder are approximately equal, indicating that the sound field is symmetrical.

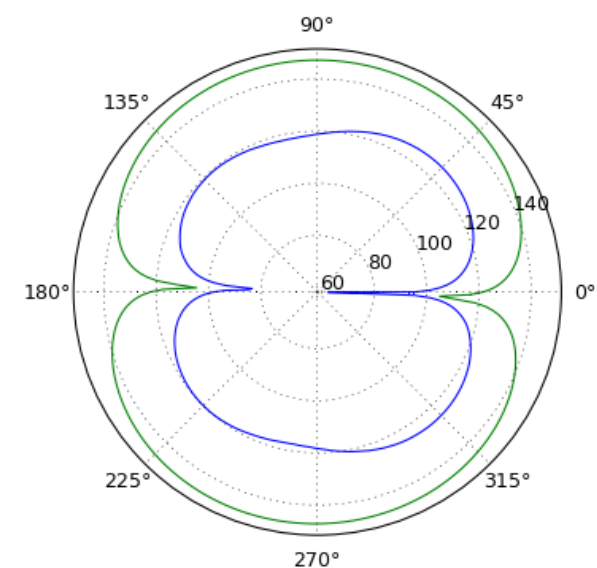

Fig. 10. Directivity of sound field of cylinder flow.

It can be seen from the directivity graph that the directivity of the radiated sound field is closest to the radiation directivity of the dipole source, indicating that the flow around the cylinder is mainly caused by the dipole formed by the unsteady pulsating pressure generated by the vortex alternately falling off the cylindrical surface.

\section{Conclusions}

In this paper, the large eddy simulation (LES), SST and k-Omega models are used to simulate the transient flow field around a three-dimensional cylinder, and the farfield radiation noise is calculated by the boundary element method. The calculation results of the example result in the following conclusions:

- LES performs better than SST and k-Omega model in transient flow simulation for cylinder flow.

- Flow noise around a cylinder is mainly concentrated in the low frequency range.

- Sound field is symmetrical perpendicular to the incoming flow direction.

- Dipole source takes the dominant place in the noise around a cylinder under the conditions of this article.

- Hybrid methods of LES and BEM can be used to simulate the flow noise of underwater structure.

The work has been supported by the China Postdoctoral Science Foundation Funded Project, No.2017M611358 and also funded by National Key Research and Development Project, No.2016YFF0200906, Heilongjiang Province Foundation, No. GX17A015. Elsewhere, the work has been supported by the major project from Acoustic Science and Technology Laboratory, No.9140C200104140C20003, and also been funded the steady support plan from Acoustic Science and Technology Laboratory (Grant No. SSJSWDZC2018005). Furthermore, the work has been funded by the project from Key Laboratory of Acoustic Stealth (Grant No.614220405011706), the Fundamental Research Funds for Central universities (Grant No.HEUCF180503), and funded by the China Scholarship Council, No.201706685061.

\section{References}

1. Revell, James D., R. A. Prydz, and A. P. Hays, Aiaa Journal, 16, 6 (1971) 
2. Brentner, Kenneth S, et al. Computational Aeroacoustics Workshop on Benchmark Problems, (1997)

3. Cox, Jared S., et al. Asme/Jsme/Imeche/ Csme/Iahr International Symposium on Fluid-structure Int (1997)

4. Osamu Inoue, Nozomu Hatakeyama, Journal of Fluid Mechanics, 471, 285-314 (2002)

5. Tang, Ke Fan, Chinese Journal of Hydrodynamics, 24,2 (2009).

6. Kravchenko, A.G., Moin. P, Physics of Fluids, 12,2(2000)

7. He H, Numerical simulation to characteristics and control of flow around a circular cylinder. BJTU, (2009) 